\title{
OCT En Face Analysis of the Posterior Vitreous Reveals Topographic Relationships among Premacular Bursa, Prevascular Fissures, and Cisterns
}

\author{
Leong, Belinda C S ; Fragiotta, Serena ; Kaden, Talia R ; Freund, K Bailey ; Zweifel, Sandrine A ;
} Engelbert, Michael

\begin{abstract}
PURPOSE: To characterize the topographic relationships among vitreous structures, including the premacular bursa, prevascular vitreous fissures, cisterns, and lacunae, in healthy participants using en face and cross-sectional swept-source (SS) OCT. DESIGN: Prospective, comparative study. PARTICIPANTS: Sixty eyes of 60 healthy participants (age range, 4-35 years). Eyes of individuals younger than 20 years $(\mathrm{n}=29)$ were compared with eyes of individuals 20 years of age or older $(\mathrm{n}=31)$. METHODS: From each study eye, $12 \times 12$-mm SS OCT volume scans comprising $1024 \times 1024$ A-scans centered at the fovea were acquired. MAIN OUTCOME MEASURES: En face and cross-sectional data were analyzed to characterize topographic relationships between hyperreflective spaces anterior to the vitreoretinal interface. RESULTS: Prevascular vitreous fissures are an almost universal feature of human eyes. Cisterns became more prevalent over the course of the first 20 years $(\mathrm{r}=0.49 ; \mathrm{P}=0.002)$. In $97 \%$ of eyes in individuals older than 20 years, en face and cross-sectional SS OCT showed the premacular bursa and prepapillary gap merge at a distance superior to the optic nerve and then follow a superonasal course anteriorly. However, only $69 \%$ of individuals younger than 20 years demonstrated such a connection $(\mathrm{P}$ $=0.01)$. A close topographic relationship of vitreous fissures and cisterns to the underlying vasculature of the posterior pole was visible on en face projections. En face imaging readily distinguished these spaces. Degenerative, eyewall-parallel fissure planes and their course were described for the first time in a 3dimensional manner. The fissure planes were rare in younger eyes (12\%) and significantly more common in older eyes $(42 \%$; $\mathrm{P}<0.001)$. CONCLUSIONS: En face SS OCT demonstrated that (1) premacular bursa and Cloquet's canal are not connected in younger patients, but are connected in older patients; (2) prevascular vitreous fissures overly the retinal vessels; and (3) cisterns are continuous with prevascular fissures.
\end{abstract}

DOI: https://doi.org/10.1016/j.oret.2019.09.002

Posted at the Zurich Open Repository and Archive, University of Zurich

ZORA URL: https://doi.org/10.5167/uzh-180162

Journal Article

Accepted Version

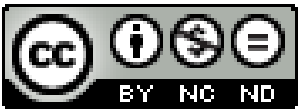

The following work is licensed under a Creative Commons: Attribution-NonCommercial-NoDerivatives 4.0 International (CC BY-NC-ND 4.0) License.

Originally published at: 
Leong, Belinda C S; Fragiotta, Serena; Kaden, Talia R; Freund, K Bailey; Zweifel, Sandrine A; Engelbert, Michael (2020). OCT En Face Analysis of the Posterior Vitreous Reveals Topographic Relationships among Premacular Bursa, Prevascular Fissures, and Cisterns. Ophthalmology Retina, 4(1):84-89.

DOI: https://doi.org/10.1016/j.oret.2019.09.002 


\section{Journal Pre-proof}

Optical coherence tomography en face analysis of the posterior vitreous reveal topographic relationships between premacular bursa, prevascular fissures and cisterns

Belinda CS. Leong, Serena Fragiotta, Talia R. Kaden, K. Bailey Freund, Sandrine

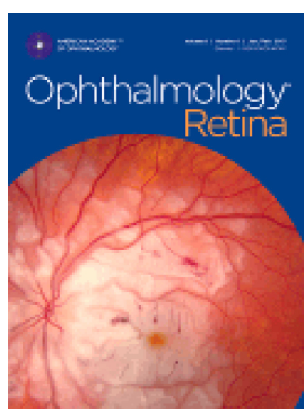
Zweifel, Michael Engelbert

PII: S2468-6530(19)30540-8

DOI: https://doi.org/10.1016/j.oret.2019.09.002

Reference: $\quad$ ORET 614

To appear in: Ophthalmology Retina

Received Date: 15 July 2019

Revised Date: 19 August 2019

Accepted Date: 3 September 2019

Please cite this article as: Leong B.C., Fragiotta S., Kaden T.R., Freund K.B., Zweifel S. \& Engelbert M., Optical coherence tomography en face analysis of the posterior vitreous reveal topographic relationships between premacular bursa, prevascular fissures and cisterns, Ophthalmology Retina (2019), doi: https:// doi.org/10.1016/j.oret.2019.09.002.

This is a PDF file of an article that has undergone enhancements after acceptance, such as the addition of a cover page and metadata, and formatting for readability, but it is not yet the definitive version of record. This version will undergo additional copyediting, typesetting and review before it is published in its final form, but we are providing this version to give early visibility of the article. Please note that, during the production process, errors may be discovered which could affect the content, and all legal disclaimers that apply to the journal pertain.

(C) YEAR Published by Elsevier Inc. on behalf of American Academy of Ophthalmology 
Optical coherence tomography en face analysis of the posterior vitreous reveal topographic relationships between premacular bursa, prevascular fissures and cisterns

Belinda CS Leong ${ }^{1,2,3}$, Serena Fragiotta ${ }^{1,2,3,4}$, Talia R. Kaden ${ }^{1,2,3,5}$, K. Bailey Freund ${ }^{1,2,3}$, Sandrine Zweifel ${ }^{6}$, Michael Engelbert ${ }^{1,2,3}$

\section{${ }^{1}$ Vitreous Retina Macula Consultants of New York, New York, USA}

${ }^{2}$ The LuEsther T. Mertz Retinal Research Center, Manhattan Eye, Ear and Throat Hospital, New York, New York, USA

${ }^{3}$ Department of Ophthalmology, New York University, New York, New York, USA

${ }^{4}$ Department of Medical-Surgical Sciences and Biotechnologies, U.O.S.D. Ophthalmology, Sapienza University of Rome, Italy

${ }^{5}$ Department of Ophthalmology, Manhattan Eye, Ear and Throat Hospital, New York, New York, USA

${ }^{6}$ Department of Ophthalmology, University of Zurich, Zurich, Switzerland

Short Title:

En Face Imaging of Vitreous Anatomy

Corresponding Author:

Michael Engelbert, MD PhD

Vitreous Retina Macula Consultants of New York

460 Park Ave. New York, NY 10022

Phone: (212) 861-9797

Fax: (212) 628-0698

E-mail address: Michael.Engelbert@gmail.com

Disclosures:

Michael Engelbert is a consultant for Genentech, Bayer, Allergan and Alimera Sciences.

K.B. Freund is a consultant to Optos, Optovue, Zeiss, Allergan, Novartis and Heidelberg Engineering and receives research support from Genentech/Roche.

Sandrine Zweifel is a consultant for Allergan, Bayer and Novartis.

The other authors have no conflicting interests to disclose.

This research was supported by a grant provided by the Macula Foundation. Serena Fragiotta was supported by Honors Center of Italian University (H2CU).

This manuscript contains two supplemental videos - Video 1 and Video 2. 
Optical coherence tomography, premacular bursa, posterior precortical vitreous pocket, vitreous,

prevascular vitreous fissure, vitreous cistern, lacuna 


\section{Abstract}

\section{Purpose:}

To characterize the topographic relationships among vitreous structures including the premacular bursa, prevascular vitreous fissures, cisterns and lacunae in healthy subjects using en face and cross-sectional swept-source optical coherence tomography (SS-OCT).

\section{Design:}

Prospective comparative study.

\section{Subjects:}

Sixty eyes of 60 healthy subjects (age range $=4-35$ years). Eyes of individuals younger than 20 years ( $n=$ 29) were compared with eyes of individuals aged 20 years or older $(n=31)$.

\section{Testing:}

Twelve $\times 12$-mm SS-OCT volume scans comprised of 1024 x 1024 A-scans centered at the fovea were acquired from each study eye.

\section{Main Outcome Measures:}

En face and cross-sectional data was analyzed to characterize topographic relationships between hyperreflective spaces anterior to the vitreoretinal interface.

\section{Results:}

Prevascular vitreous fissures are an almost universal feature of human eyes. Cisterns became more prevalent over the course of the first 20 years $(r=0.49, P=0.002)$. In $97 \%$ of eyes in individuals older than 20, en face and cross-sectional SS-OCT showed the premacular bursa and prepapillary gap merge at a distance superior to the optic nerve and then follow a superonasal course anteriorly. However, only $69 \%$ of individuals younger than 20 years demonstrated such a connection $(p=0.01)$. A close topographical relationship of vitreous fissures and cisterns to the underlying vasculature of the posterior pole was visible on en face projections. En face imaging readily distinguishes these spaces. Degenerative, eyewall- 
66 parallel fissure planes and their course are described for the first time in a three-dimensional manner.

67 The fissure planes were rare in younger eyes (12\%), and significantly more common in older eyes (42\%;

$68 p<0.001)$.

69 Conclusions:

70 En-face SS-OCT demonstrates (1) that premacular bursa and Cloquet's canal are not connected in

71 younger, but in older patients, that (2) prevascular vitreous fissures overly the retinal vessels, and (3)

72 that cisterns are continuous with PVFs. 


\section{Introduction}

Although the vitreous comprises the majority of intraocular volume, our understanding of its normal anatomy and pathologic changes lags behind that of many chorioretinal structures. Technical limitations related to imaging a nearly optically clear structure have made study of the vitreous particularly challenging. Post-mortem histologic studies of the vitreous are limited by high water content, producing numerous post-fixation artifacts.

Initial ex vivo studies of the vitreous by Worst identified the premacular bursa in $1977^{1}$, with further refinements made by Jongebloed and Worst in $1987^{2}$ and Kishi and Shimizu in $1990^{3}$. Kishi renamed this structure the "posterior precortical vitreous pocket" (PPVP) ${ }^{3}$ to emphasize the presence of cortical vitreous fibers at its posterior border. Eisner discovered "prevascular fissures" (PVF) when he noted hyporeflective, presumably liquid vitreous structures when analyzing cadaveric eyes . ${ }^{4,5}$ Cisterns were noted a few years later by Jongebloed and Worst ${ }^{2}$ as a system of "hollow" spaces.

The advent of optical coherence tomography (OCT) allowed for unprecedented in vivo visualization of the formed vitreous and the liquid spaces it contains. OCT confirmed the presence of the premacular bursa and other vitreous structures previously reported in cadaver eyes. ${ }^{6}$

In this study, optimized en face SS-OCT was utilized to visualize the vitreous structure in an effort to resolve lingering controversies and ambiguities from prior studies that either relied on cross-sectional OCT or en face OCT with lower resolution. Based on prior cross-sectional sampling of the posterior vitreous with SS-OCT it was hypothesized that hyporeflective spaces overlying the large vessels of the posterior pole follow an uninterrupted course over these vessels and that as one moves anteriorly in the eye and further from the retinal surface, these spaces form cisterns in the context of vitreous 
degeneration. The relationship between the premacular bursa and prepapillary gap (defined as the space originating from the area of Martegiani overlying the optic nerve head; often termed Cloquet's canal) was also examined more closely. In order to distinguish liquid spaces apart from the premacular bursa, prepapillary gap and PVFs, the term "cistern" was used to define empty vitreous spaces in connection with fissures, and "lacunae" was used to refer to optically empty spaces that are distinctly separate from PVFs.

\section{Methods}

Our study adhered to the Tenets of the Declaration of Helsinki on the ethical principles of medical research and complied with the Health Insurance Portability and Accountability Act of 1996. It was approved by Western Institutional Review Board (IRB) committee, as well as the Ethics committee of the Canton of Zurich, Switzerland. Written informed consent was obtained from each subject or, in the case of minors, a parental guardian.

\section{Healthy subjects with no known anterior segment or vitreoretinal pathology were prospectively enrolled} in this study. Patients were divided into two groups according to age. The younger group $(<20$ years old) ranged from age 4 to 19 years, whereas the older group ( $\geq 20$ years old) ranged from 22 to 35 -yearsold.

Vitreous imaging was performed on at least one eye of each subject, based upon individual preference and ease of acquisition. When both eyes were imaged, the eye with the higher quality acquisition was chosen for analysis. Otherwise, the right eye was typically scanned. 
All eyes were imaged on the PLEX Elite 9000 (Carl Zeiss Meditec Inc., Dublin, CA) optical coherence tomography device. The PLEX Elite 9000 uses a swept-source laser with a center wavelength of 1040$1060 \mathrm{~nm}$ and scan speed of 100000 A-scans per second. Cube $(12 \mathrm{~mm} \times 12 \mathrm{~mm})$ scans comprised of 1024 B-scans (each comprising 1024 A-scans) and centered at the fovea were obtained for each study eye. During scan acquisition, visualization of vitreous structures was enhanced by positioning the retina and choroid posteriorly within the scan window. In 17 eyes, single highly averaged (100 frames) $16 \mathrm{~mm}$ horizontal and vertical B-scans were obtained. In most of the patients older than 20 years, axial length measurements could be obtained, but this was not possible in the majority of subjects in the younger age group.

\section{Image analysis}

En face structural slabs of the vitreoretinal interface were created from the $12 \times 12-\mathrm{mm}$ cube scans by selecting the automated ILM segmentation as the posterior boundary and $-150 \mu \mathrm{m}$ as the anterior boundary. A dynamic assessment of these images was performed by manually adjusting the slab thickness (range $=80-150 \mu \mathrm{m}$ ) and its distance from the retinal surface, which enabled a 3-dimensional en face assessment of the vitreoretinal interface (Figure 1, movie). Brightness and contrast of both the en face and cross-sectional views were adjusted to optimize visualization of vitreous anatomy.

During the dynamic assessment, the greatest horizontal diameter of the premacular bursa was measured and the presence or absence of communication between the premacular bursa and prepapillary gap was noted (Figure 2). When present, the distance from the temporal edge of the optic disc to the communication was measured. For each eye, the presence or absence of PVF, cisterns and lacunae were recorded. Prevascular fissures were defined as slender, hyporeflective vitreous cavities overlying retinal vessels. Cisterns were defined as larger hyporeflective spaces that could be traced posteriorly to an associated retinal vessel. In contrast, lacunae were defined as hyporeflective vitreous 
spaces with no identifiable association with a specific retinal vessel, and no visible connection to a PVF or prevascular cisterns.

\section{Statistical analysis}

Quantitative variables were expressed as mean \pm standard deviation (SD) and the normality of distribution was verified through Shapiro-Wilk normality test. Standard error (SE) was used instead of SD in cases of non-parametric distribution (Shapiro-Wilk, $\mathrm{P}<0.05)$. Chi-squared test was used to compare categorical variables, whereas Spearman's rank correlation or Kendall's tau coefficient were calculated to determine the relationship between variables. $P$ values less than 0.05 were considered statistically significant, and $\mathrm{P}<0.001$ considered highly statistically significant. All calculations were carried out using SPSS software (ver.20; SPSS, Inc., Chicago, IL).

\section{Results}

A total of 60 eyes of 60 healthy subjects (age range: $4-35$ years) were included in the present study and divided into two age cohorts ( $<20$ years old; $\geq 20$ years old). The main characteristics of the two cohorts are summarized in Table 1.

\section{Prevascular vitreous fissures}

Prevascular vitreous fissures follow a continuous course over the vascular arcades on Z-scan (Figure 1) in all subjects.

The presence of cisterns is associated with age and speckling in children and young adolescents

Cisterns are wider spaces than PVFs, but are found in the same location over the vascular arcades. In the younger cohort, the presence of cisterns was significantly associated with increasing age $(r=0.49$, 
$P=0.002$ ), with an average likelihood of $72 \%$. In the older cohort, no association with increasing age could be found ( $r=0.17, P=0.39)$, and average likelihood was $80 \%$ (Table 2 ).

The presence of cisterns was also directly and strongly related to the presence of speckling surrounding the cisterns $(r=0.81, \mathrm{P}<0.001)$ in both cohorts.

\section{Premacular bursa and prepapillary gap connect with increasing age}

The maximal width of the premacular bursa did not differ significantly between age-groups (mean difference: $-276.9 \mu \mathrm{m}, \mathrm{Cl} 95 \%:-901.5,347.7 \mu \mathrm{m}, \mathrm{P}=0.38)$, as reported in Table 1. The likelihood of a connection between premacular bursa and prepapillary gap was 1.50 times higher $\left(\chi^{2}: 8.38, P=0.01\right)$ in the older cohort relative to the younger subgroup (Table 2). The presence of a connection between the premacular bursa and the peripapillary gap in the overall population (younger and older cohorts) was positively correlated with age $(r=0.28, P=0.03)$.

\section{Fissure planes can be seen with increasing age}

During data analysis, it was apparent that a structure different from the typical oblate spheroidal lacunae in the central vitreous could be seen in addition to, and connected with, PVFs and cisterns.

These "fissure planes" are comparatively thin and wide, undulating in their course and show speckled hyperreflectivity at their edges (Figure 3). While younger eyes showed a relatively homogeneous gel vitreous bordering the premacular bursa, PVFs and cisterns, older eyes demonstrated significantly more frequent additional spaces. The likelihood of fissures planes was 4.33 times greater in the older cohort than the younger subgroup $\left(\chi^{2}: 14.34, P=0.001\right.$, Table 2$)$ and correlated with increasing age $(r=0.32$, 
$\mathrm{P}=0.003)$. In the older cohort, presence of fissure planes increase with the presence of cisterns and speckling ( $r=0.42, P=0.02$ for both), as either structure would arise from the PVF accordingly.

The presence of cisterns and speckling is related with gender in young adults

The presence of cisterns was moderately associated with female gender $(r=0.46, P=0.02)$ in young adults. The cisterns were recognized in 19 of 21 eyes (90.5\%) of females included in the older subgroup, whereas the males exhibited cisterns in only 6 of 10 eyes $(60 \%, P=0.03)$. Similarly, the presence of speckling was detected with the same frequencies among females (19 of 21 eyes, 90.5\%) and males (6 of 10 eyes, $60 \%, \mathrm{P}=0.03)$ in the older cohort.

\section{Discussion}

The data presented herein put to rest the vexing question of how the posterior liquid spaces of the vitreous relate to one another. Kishi has postulated that the PPVP is a confined, boat-shaped space with at most, a thin connecting channel to "Cloquet's canal". 6,18,19 In contrast, Worst maintained that these spaces were separated by the "septum interpapillomaculare," and that rather than being a confined space, the "premacular bursa," as he called it, was connected to far more anterior vitreous spaces. ${ }^{2,7,12}$ In vivo study has recently become feasible with the advent of OCT, but even then, this work has been limited by resolution and scan volume.$^{6,13-22}$ Current SS-OCT technology has made it possible not only to scan both young children and adults in a fraction of the time, but with unprecedented resolution, volume and sampling density.

Our study demonstrates that Kishi's view of a confined space appears accurate in some subjects on the younger end of our cohort, where we found that the premacular bursa and area of Martegiani were not 
connected. However, our wide scans also demonstrated that one key aspect of Worst's concept of the "bursa" holds true: the bursa does not have a natural anterior border. Even our youngest subjects showed an anterior and superior extension of the bursa that reached beyond the scanned volume of 12 x $12 \times 3 \mathrm{~mm}$. On the other hand, we observed one young adult with a "dollop"-shaped phenotype where the bursa points forward toward the center of the vitreous, and the superior and inferior aspect of the bursa's border appear to meet. This supports Eisner's hypothesis that the liquid vitreous spaces overlying the posterior pole are the result of a non-production of vitreous over the optic nerve (the area of Martegiani), and relatively decreased production in the central macula due to the process of foveation and consequent lateral displacement of nascent vitreous. Notably, this also explains the absence of formed vitreous over the blood vessels, thus giving rise to PVFs. By employing our Z-scan visualization method, we were able to demonstrate the continuous course of these fissures for the first time. All of these spaces are expected to open into the more anterior vitreous, making them more pocket-like.

We have previously hypothesized the presence of PVF and cisterns in vivo based on limited crosssectional OCT sampling. ${ }^{23}$ We also postulated that the PVFs are precursors to cisterns, since the base of both structures appeared to overlie superficial retinal blood vessels, and there was a trend towards an increasing prevalence of cisterns with advancing age, while the prevalence of PVFs declined. ${ }^{23}$ It has been suggested that the development of PVFs results from a lack of hyalocytes overlying the retinal blood vessels. ${ }^{5,23}$ Subsequent syneresis may then lead to the expansion of the PVFs, eventually forming cisternal spaces. Vitreous degeneration may also be responsible for the connection of the premacular bursa and area of Martegiani, as well as the formation of fissure planes. We have previously reported the presence of "fissure planes" as undulating liquid spaces with irregular, "speckled" edges anterior to the premacular bursa and associated them with increasing vitreous degeneration. ${ }^{23}$ In the present study, concurrent en face and cross-sectional analysis enabled a three-dimensional characterization of 
these structures. Fissure planes were found significantly more often with greater fissure width across the entire superior and/or inferior temporal retinal vessel arcades.

"Speckling" has been observed by ourselves and others on cross-sectional vitreous OCT images and is thought to correspond to the aggregation of collagen fibrils supporting the primarily water-based structure. ${ }^{11,20,23}$ Vitreous fibers comprising the posterior hyaloid were noted by Liu and colleagues in their en face analysis. ${ }^{24}$ It has already been reported that this fibrillar structure contains gaps where PVFs occur. ${ }^{25}$ We postulate that progressive vitreous syneresis leads to expansion of liquefied spaces, and that fibrils would be further pushed aside and aggregate to form irregular tangles at the edges of cisterns or fissure planes. This was inferred from cross-sectional analysis, but can now be clearly seen in high resolution en face images (Figure 3). We were able to observe that all cisterns were surrounded by speckling. It will be interesting to assess development of this morphologic pattern in a cohort with a wider age range as we show a correlation between the presence of PVFs with speckling and cisterns. There are several important limitations to this study. Current imaging technology is unable to provide visualization of more central vitreous, and this makes it impossible to follow the liquid spaces more anteriorly. Furthermore, vitreous degeneration is likely not only a result of aging. However, the subject numbers obtainable in studies such as these make it difficult to assess what role variables such as axial length, gender and maybe even ethnicity may play in vitreous anatomy.

In summary, Z-scans obtained with the latest SS-OCT technology allow us to lift the shroud that has veiled the "enigmatic" vitreous and to lay to rest some of the controversies surrounding its liquid spaces. In recognition of Kishi's and Worst's contributions we suggest renaming the PPVP or premacular bursa the "premacular pocket". We also suggest renaming what many call Cloquet's canal, as the area of Martegiani. The empty space overlying the optic nerve is very probably the result of absent vitreous 
production, rather than the contents of the hyaloid vasculature. The basement membrane remnant of the hyaloid vasculature has probably long disintegrated, possibly manifesting only as the hyperreflective strands in proximity to the area of Martegiani.

262 The pathogenetic contribution of the vitreous gel and the liquid structures contained within the posterior hyaloid membrane is likely underappreciated, but advancements in OCT technology may herald a new era of discovery, hopefully enhancing our management of vitreoretinal disorders. 
1. Worst JG. Cisternal systems of the fully developed vitreous body in the young adult. Trans Ophthalmol Soc U K 1977.

2. Jongebloed WL, Worst JFG. The cisternal anatomy of the vitreous body. Doc Ophthalmol 1987;67:183-196.

3. Kishi S, Shimizu K. Posterior Precortical Vitreous Pocket. Curr Eye Res 1990;108:979-982.

4. Eisner G. Biomicroscopy of the peripheral fundus. New York, NY: Springer-Verlag; 1979.

5. Eisner G. Clinical anatomy of the vitreous. In: Jacobiec F, ed. Ocular Anatomy, Embryology, and Teratology. Philadelphia, PA: Harpe \& Row Publishers; 1982:391-424.

6. Itakura H, Kishi S, Li D, Akiyama H. Observation of posterior precortical vitreous pocket using sweptsource optical coherence tomography. Investig Ophthalmol Vis Sci 2013;54:3102-3107.

7. Worst JG. Posterior precortical vitreous pocket. Arch Ophthalmol 1991;109:1058-1059.

8. Denlinger JL, Eisner G, Balazs E a. Age-related changes in the vitreus and lens of rhesus monkeys 278 (Macaca mulatta). Exp Eye Res 1980;31:67-79. Available at:

279 http://www.ncbi.nlm.nih.gov/pubmed/7428838.

9. Kishi S, Shimizu K. Posterior precortical vitreous pocket [reply to letter]. Arch Ophthalmol 1991;109:1060.

10. Sebag J. Posterior precortical vitreous pocket. Arch Ophthalmol 1991;109:1059-1060.

11. Sebag J. Age-related changes in human vitreous structure. Graefe's Arch Clin Exp Ophthalmol 1987;225:89-93.

12. Worst JG. Cisternal systems of the fully developed vitreous body in the young adult. Trans Ophthalmol Soc U K 1977;97:550-4.

13. Itakura H, Kishi S. Aging changes of vitreomacular interface. Retina 2011;31:1400-1404. 
Ophthalmol 2013;131:1095-1096. Available at: http://www.ncbi.nlm.nih.gov/pubmed/23765255. 15. Schaal KB, Pang CE, Pozzoni MC, Engelbert M. The Premacular Bursa's Shape Revealed In Vivo by Swept-Source Optical Coherence Tomography. Ophthalmology 2014;121:1020-1028. Available at: http://dx.doi.org/10.1016/j.ophtha.2013.11.030.

16. Itakura H, Kishi S. Evolution of vitreomacular detachment in healthy subjects. JAMA Ophthalmol 2013;131:1348-1352.

17. Itakura H, Kishi S, Li D, et al. Vitreous changes in high myopia observed by swept-source optical coherence tomography. Investig Ophthalmol Vis Sci 2014;55:1447-1452.

18. Itakura H, Kishi S, Li D, Akiyama H. En face imaging of posterior precortical vitreous pockets using swept-source optical coherence tomography. Investig Ophthalmol Vis Sci 2015;56:2898-2900.

19. Li D, Kishi S, Itakura H, et al. Posterior precortical vitreous pockets and connecting channels in children on swept-source optical coherence tomography. Investig Ophthalmol Vis Sci 2014;55:24122416.

20. Spaide RF. Visualization of the posterior vitreous with dynamic focusing and windowed averaging swept source optical coherence tomography. Am J Ophthalmol 2014;158:1267-1274.

21. Park KA, Oh SY. Posterior Precortical Vitreous Pocket in Children. Curr Eye Res 2015;40:1034-1039.

22. Stanga PE, Sala-Puigdollers A, Caputo S, et al. In vivo imaging of cortical vitreous using 1050-nm swept-source deep range imaging optical coherence tomography. Am J Ophthalmol 2014;157.

23. Pang CE, Schaal KB, Engelbert M. Association of prevascular vitreous fissures and cisterns with vitreous degeneration as assessed by swept source optical coherence tomography. Retina $2015 ; 35: 1875-1882$.

24. Liu JJ, Witkin AJ, Adhi M, et al. Enhanced vitreous imaging in healthy eyes using swept source optical coherence tomography. PLoS One 2014;9:1-10.

25. Gal-Or O, Ghadiali Q, Dolz-Marco R, Engelbert M. In vivo imaging of the fibrillar architecture of the 
posterior vitreous and its relationship to the premacular bursa, Cloquet's canal, prevascular vitreous fissures, and cisterns. Graefe's Arch Clin Exp Ophthalmol 2019;257:709-714.

26. Mojana F, Kozak I, Oster SF, et al. Observations by Spectral-Domain Optical Coherence Tomography Combined with Simultaneous Scanning Laser Ophthalmoscopy: Imaging of the Vitreous. Am J Ophthalmol 2010;149:641-650.

27. Kishi S, Hagimura N, Shimizu K. The role of the premacular liquefied pocket and premacular vitreous cortex in idiopathic macular hole development. Am J Ophthalmol 1996;122:622-628.

28. Spaide RF. Measurement of the posterior precortical vitreous pocket in fellow eyes with posterior vitreous detachment and macular holes. Retina 2003;23:481-485. 
Figure Legends:

Figure 1:

En face scans at 141,365 and $1250 \mu \mathrm{m}$ respectively anterior to the retinal surface $(\mathrm{A}-\mathrm{C})$ demonstrate a relatively well-preserved vitreous structure in a 24-year-old female with an axial length of $24.20 \mathrm{~mm}$. The black arrows indicate prevascular vitreous fissures (A) while the white arrows (C) indicate cisterns more anteriorly. Both the bursa (A, asterisk) and the prepapillary gap/area of Martegiani ( $A$, white triangle) can be appreciated in en face scans.

En face scan of a 34-year-old with an axial length of $25.96 \mathrm{~mm}$ (D-F) demonstrates increasing vitreous syneresis at 86, 365 and $950 \mu \mathrm{m}$ respectively anterior to the retinal surface. Both fissures (black arrows) and cisterns (white arrows) are present in increasing number and size. The black triangle indicates a degenerative fissure plane with surrounding aggregated vitreous fibrils (open arrow).

\section{Figure 2:}

$16 \mathrm{~mm}$ vertical scans from the same subject as in Figure $1 \mathrm{~A}-\mathrm{C}$ show an anteriorly pointed "dollop" configuration of the pre-macular bursa (A, asterisk). In contrast, a more flattened, elongated, and superiorly directed bursa is present in the subject from Figure $1 \mathrm{D}-\mathrm{F}$ (B, asterisk). A degenerative fissure plane is seen ( $B$, black triangle), as well as speckling in proximity ( $B$, open arrow).

Figure 3:

En face scan $789 \mu \mathrm{m}$ anterior to the retinal surface from the same subject as in Figure D-F demonstrates speckling ( $A$, white arrows) at the edge of cisterns and the degenerative fissure plane ( $A, B$; black triangles). The green line $(A)$ represents the cross-sectional slice seen in $B$. B demonstrates the vitreoretinal interface segmentation (purple lines) used to create the en face image seen in A with speckling seen in this scan as well (white arrows). The overexposure of the retina is a result of the increased brightness required to visualize the vitreous. 
Video 1:

350 A video scrolling through the $12 \times 12 \mathrm{~mm}$ en face scans from the 24 -year-old female whose images are reported in greater detail in Figure 1; A-C and Figure 2A. This video clearly illustrates the prevascular fissures, cisterns, bursa and area of Martegiani and their relationship to one another.

\section{Video 2:}

354 A video scrolling through the $12 \times 12 \mathrm{~mm}$ en face scans from the 34 -year-old female whose images are reported in Figure 1; D-F and Figure 2B. When compared with Video 1, this video illustrates increased vitreous syneresis, with larger and more numerous fissures and cisterns. The degenerative fissure plane and surrounding coiled vitreous fibrils seen clearly in Figure 1F are also well appreciated here. 
Table 1. Demographics and characteristic of the populations.

\begin{tabular}{lccc}
\hline & $\begin{array}{c}\text { Younger } \\
(<20 \text { years old })\end{array}$ & $\begin{array}{c}\text { Older } \\
(\geq 20 \text { years old })\end{array}$ & P \\
\hline Total no. of eyes & 26 & 31 & - \\
Age, mean \pm SD & $9.6 \pm 3.6$ & $28 \pm 4$ & $<0.001$ \\
Gender, Female, N (\%) & $13(50)$ & $21(67.7)$ & 0.14 \\
$\begin{array}{l}\text { Premacular bursa maximal width } \\
(\mu \mathrm{m})\end{array}$ & $6019.2 \pm 1161.3$ & $6209 \pm 1286.7$ & 0.28 \\
\hline
\end{tabular}

SD: standard deviation; N: number; \%: percentage; P values were calculated with unpaired ttest for quantitative variables and Chi-squared was used to compare qualitative variables. 
Table 2. Morphometric characteristics of vitreous cavity between children/young adolescents and young adults.

\begin{tabular}{lccc}
\hline & $\begin{array}{c}\text { Younger } \\
(<20 \text { years old })\end{array}$ & $\begin{array}{c}\text { Older } \\
(\geq 20 \text { years old })\end{array}$ & P \\
\hline Total no. of eyes & 26 & 31 & \\
\hline Presence of, N (\%) & & $31(100)$ & 0.46 \\
Fissures overlying vessels & $25(96.2)$ & $25(80.6)$ & 0.25 \\
Cisterns overlying vessels & $18(69.2)$ & $30(96.8)$ & $\mathbf{0 . 0 0 2}$ \\
Connection & $17(65.4)$ & $13(41.9)$ & $<0.001$ \\
Fissures planes & $3(11.5)$ & $25(80.6)$ & 0.49 \\
$\begin{array}{l}\text { Speckling } \\
\text { SD: standard deviation; N: number; \%: percentage; }{ }^{*} \text { Connection between premacular bursa } \\
\text { and peripapillary gap; P value was obtained using Chi-squared test. }\end{array}$ & \\
\hline
\end{tabular}




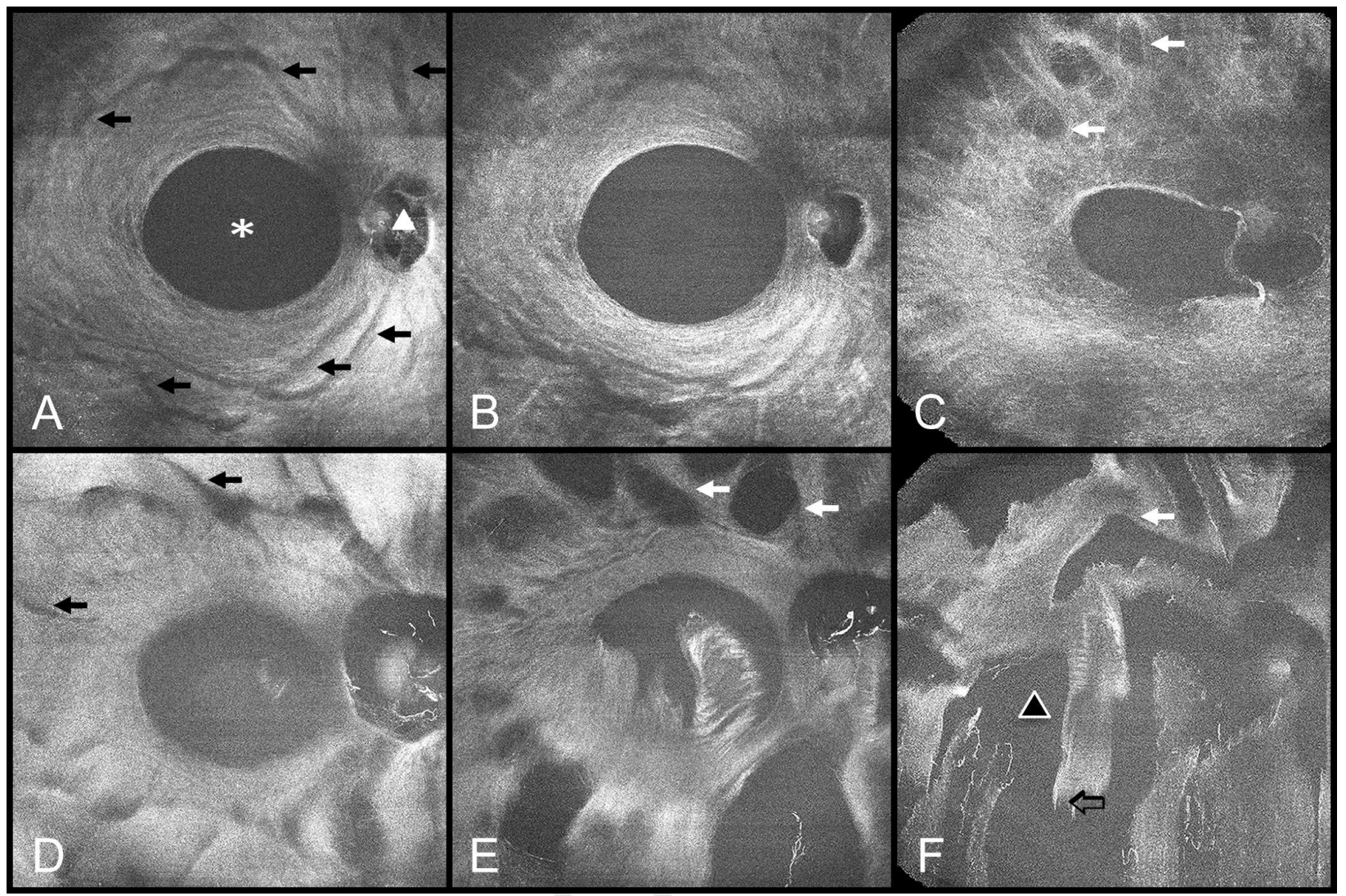




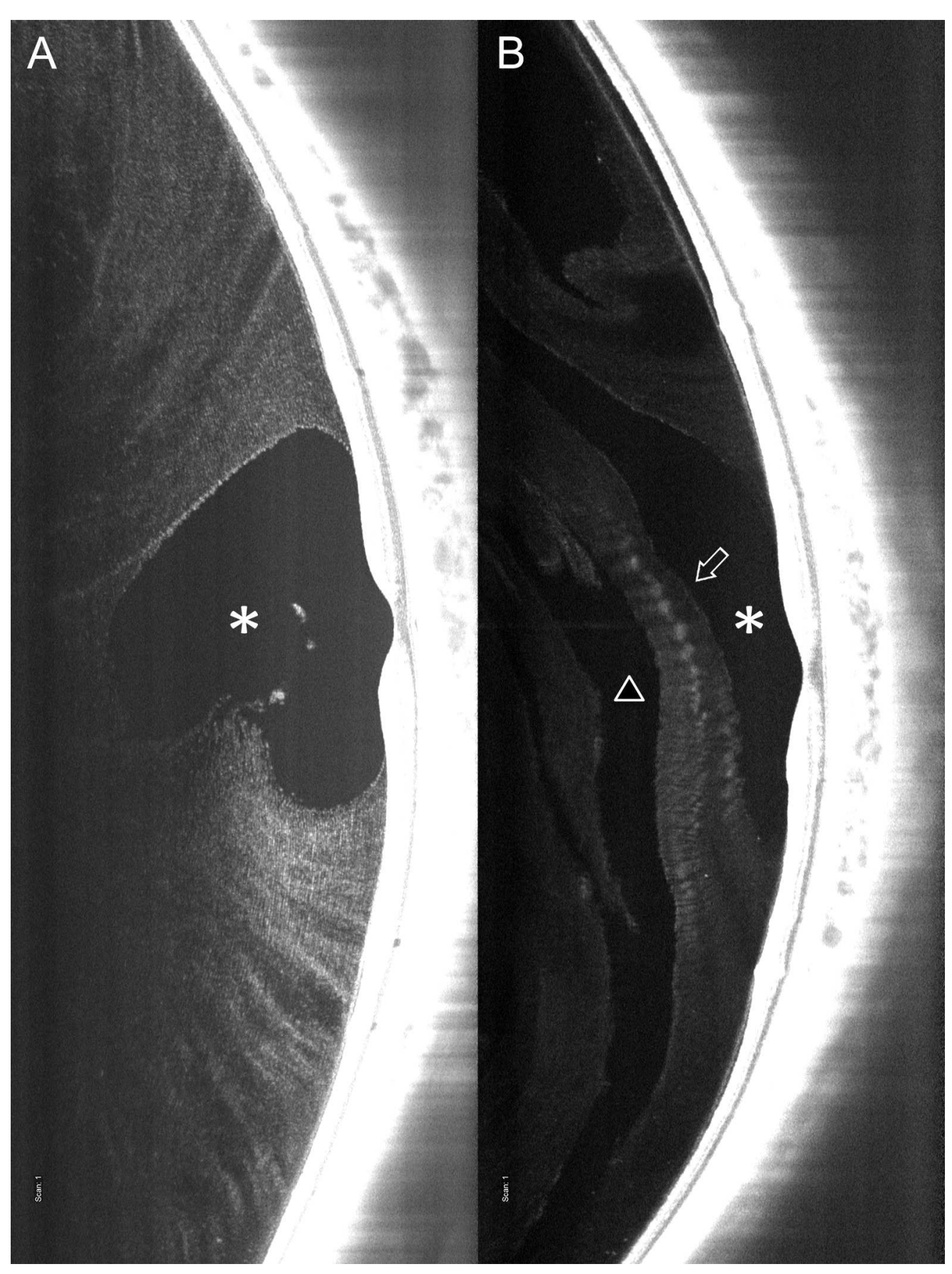




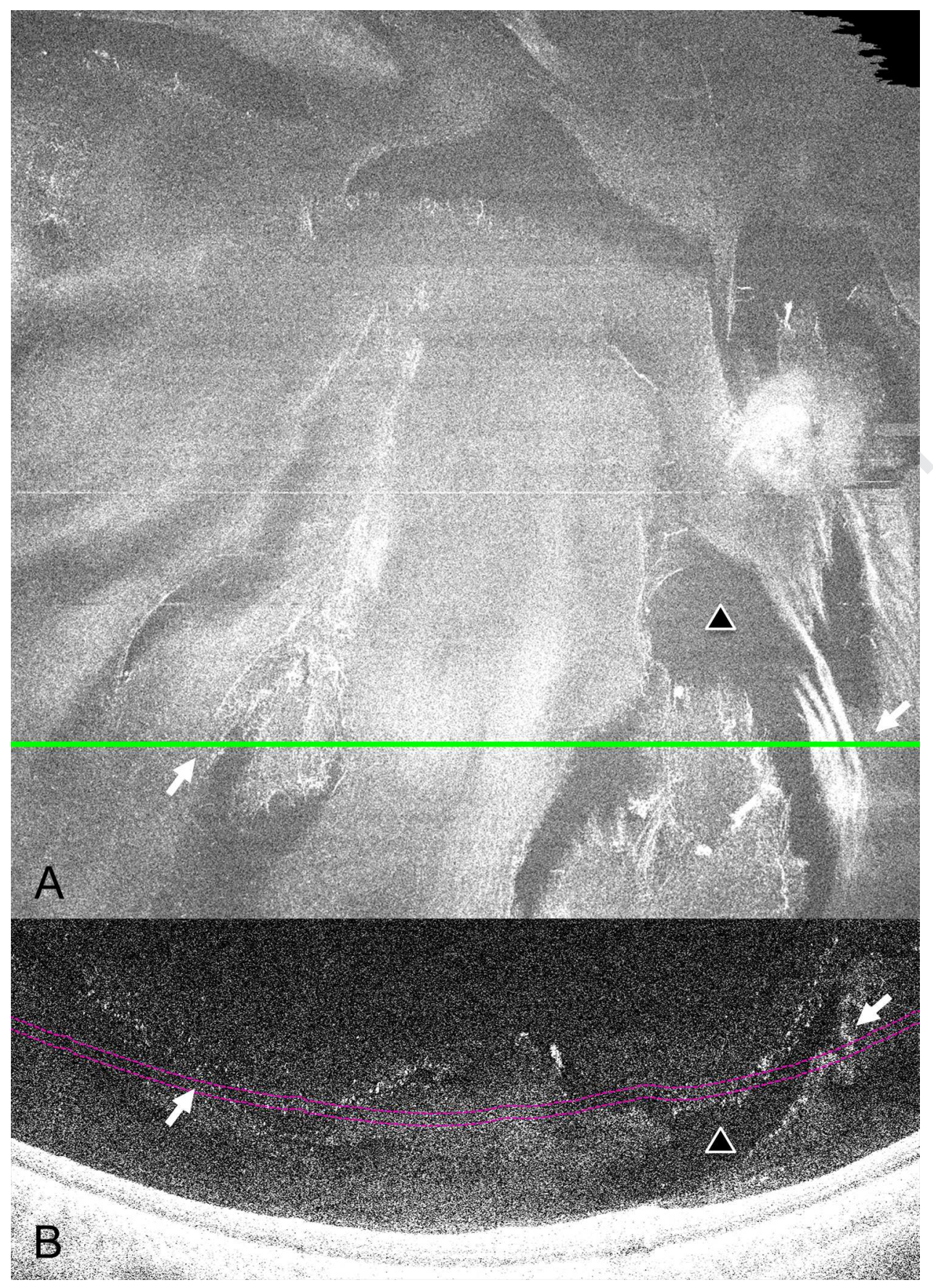


SS-OCT demonstrates (1) that premacular bursa and Cloquet's canal are not connected in younger, but in older patients, that (2) prevascular vitreous fissures overlie the retinal vessels, and (3) that cisterns are continuous with PVFs. 